\title{
AN EXAMPLE IN THE FIXED POINT THEORY OF POLYHEDRA ${ }^{1}$
}

\author{
BY WILLIAM LOPEZ
}

Communicated by Felix Browder, May 29, 1967

1. Introduction. A finite polyhedron is constructed which enables us to answer the following questions in the negative.

(1) Is the fixed point property a homotopy type invariant in the category of finite polyhedra?

(2) Is the fixed point property a product invariant in the category of finite polyhedra, i.e. if $K_{1}$ and $K_{2}$ have the fixed point property, does $K_{1} \times K_{2}$ have the fixed point property?

The author is indebted to Professor Edward Fadell for bringing these questions to his attention, and pointing out that if one found a polyhedron with the fixed point property and yet admitted a map with even Lefschetz number, then these questions could be answered.

2. The example. Let

$$
X=P_{2}(C) \cup S_{1} \times S_{2} \cup P_{4}(C)
$$

where $P_{2}(C)$ and $P_{4}(C)$ are complex projective spaces, $S_{1}$ and $S_{2}$ are 2-spheres, and the following identifications are made. Letting $\left(b_{1}, b_{2}\right) \in S_{1} \times S_{2}$ be a base point, $P_{1}(C) \subset P_{2}(C)$ is identified with $S_{1} \times b_{2}$ and $P_{1}(C) \subset P_{4}(C)$ is identified with $b_{1} \times S_{2}$.

The cohomology ring structure of $X$ over the rational field $Q$ is given by:

$$
\begin{array}{ll}
H^{0}(X ; Q)=Q, & \text { with generator } 1, \\
H^{2}(X ; Q)=Q \oplus Q, & \text { with generators } \alpha, \beta, \\
H^{4}(X ; Q)=Q \oplus Q \oplus Q, & \text { with generators } \alpha^{2}, \alpha \beta, \beta^{2}, \\
H^{6}(X ; Q)=Q, & \text { with generator } \beta^{3}, \\
H^{8}(X ; Q)=Q, & \text { with generator } \beta^{4} .
\end{array}
$$

All odd cohomology is zero, and furthermore, $\alpha^{3}=\alpha^{4}=\alpha \beta^{2}=\alpha^{2} \beta=0$. Note that $\chi(X)=8$. ( $\chi$ denotes Euler characteristic.)

TheOREM 1. $X$ has the fixed point property.

\footnotetext{
1 This research was supported by the National Science Foundation under Grant GP-6682 and constitutes part of the author's dissertation written under the direction of Professor Edward Fadell.
} 
Proof. Let $f: X \rightarrow X$ denote any map and suppose $f^{*}(\alpha)=a \alpha+b \beta$, $f^{*}(\beta)=c \alpha+d \beta$. Let $g$ denote the composite

$$
P_{4}(C) \stackrel{i}{\rightarrow} X \stackrel{f}{\rightarrow} X \stackrel{r}{\rightarrow} P_{2}(C)
$$

where $r$ is the retraction which sends $S_{1} \times S_{2}$ onto $S_{1} \times b_{2}$ and $P_{4}(C)$ onto $\left(b_{1}, b_{2}\right)$. If $\alpha_{1} \in H^{2}\left(P_{2}(C)\right)$ and $\beta_{1} \in H^{2}\left(P_{4}(C)\right)$ are generators, then $g^{*}\left(\alpha_{1}\right)=b \beta_{1}$ and $g^{*}\left(\alpha_{1}^{3}\right)=b^{3} \beta_{1}^{3}=0$. Thus $b=0$ and hence the Lefschetz number of $f$ is given by

$$
\begin{aligned}
L(f) & =1+a+d+a^{2}+a d+d^{2}+d^{3}+d^{4} \\
& =\left(a+\frac{1}{2}+d / 2\right)^{2}+\frac{1}{4}\left(4 d^{4}+4 d^{3}+3 d^{2}+2 d+3\right) .
\end{aligned}
$$

If we let

$p(d)=4 d^{4}+4 d^{3}+3 d^{2}+2 d+3, \quad p^{\prime}(d)=2(2 d+1)\left(4 d^{2}+d+1\right)$

and we see that $p(d) \geqq p(-1 / 2)=5 / 2$ and hence $L(f)>0$.

3. Consequences. We first recall a theorem of Wecken [3, Theorem 2] which may be stated, in part, as follows:

TheOREM W. Let $K$ be a finite polyhedron with the property that no finite collection of points separates $K$. Then $K$ admits a fixed point free map (homotopic to identity) if $\chi(K)=0$.

Let $Y=\Sigma P_{8}(C)$ be the suspension of complex projective 8-space. A simple argument using Steenrod squares shows that $Y$ has the fixed point property. Since $\chi(Y)=-7, \chi(X \vee Y)=0$.

ThEOREM 2. $X \bigvee Y$ is a finite polyhedron of Euler characteristic 0 with the fixed point property.

Theorem 3. $X$ and $Y$ are two finite polyhedra with the fixed point property such that their union along an edge fails to have the fixed point property.

Just apply Theorem $\mathrm{W}$ to $X \cup_{I} Y$, the union of $X$ and $Y$ joined along an edge.

Since $X \bigvee Y$ and $X \cup_{I} Y$ are of the same homotopy type, we have the following:

CoRollary. The fixed point property is not a homotopy type invariant in the category of finite polyhedra.

Now, let $Z=X \vee Y$. Applying Theorem $\mathrm{W}$ to $Z \times I$ and $Z \times Z$ we obtain 
THEOREM 4. $Z$ is a polyhedron with the fixed point property such that $Z \times I$ and $Z \times Z$ fail to have the fixed point property. Thus the fixed point property is not a product invariant in the category of finite polyhedra.

Note that $\chi\left(\Sigma^{2} Z\right)=0$ and hence, using Theorem $W$ again, the double suspension $\Sigma^{2} Z$ admits a fixed point free map. $\Sigma Z$ either admits a fixed point free map or has the fixed point property. In either case, we obtain

THEOREM 5. There is a finite polyhedron $K$ with the fixed point property such that $\Sigma K$ fails to have the fxed point property.

Remarks. Note that all the above examples are simply connected.

We might also mention that, using [1] and [2], the fixed point property is a homotopy type invariant in the category of polyhedra of $\operatorname{dim}>2$ having the homotopy type of simply connected, closed topological manifolds.

\section{REFERENCES}

1. E. Fadell, On a coincidence theorem of F. B. Fuller, Pacific J. Math. (3) 15(1965), 825-834.

2. Shi Gen-Hua, On least number of fixed points and Nielsen numbers, Chinese Math. 8 (1966), 234-243.

3. Wecken, Fixpunktklassen. III, Math. Ann. 118-119 (1941-1943), 544-577.

UNIVERSITY OF WISCONSIN 